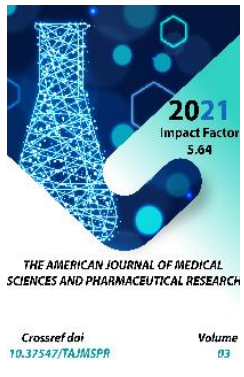

Copyright: Original content from this work may be used under the terms of the creative commons attributes 4.0 licence.

\section{Diagnosis And Treatment Of Emphysematous Pyelonephritis In Patients With Diabetes}

\author{
Nurillo Makhmudov \\ Docent, Department Of Internal Medicine, Fergana Medicine Institute, Uzbekistan \\ Zukhra Kosimova \\ Assistant Docent, Department Of Internal Medicine, Fergana Medicine Institute, Uzbekistan
}

\title{
ABSTRACT
}

In this study we report on our experience in treating two patients diagnosed with a rare disease of emphysematous pyelonephritis, who were admitted to the Urology department of Fergana Medical Institute during 2018. We provide details on each test conducted on patients, pre- and post-surgery. Our analysis shows that to prevent complications in patients with diabetes mellitus, dispensary observation with control of glucose levels, urine and blood tests is necessary.

\section{KEYWORDS}

Emphysematous Pyelonephritis, Diabetes.

\section{INTRODUCTION}

Emphysematous pyelonephritis is a severe, lifethreatening disease characterized by the presence of renal parenchymal and perirenal infections caused by gaseous microorganisms (such as E. coli). Mostly emphysematous pyelonephritis occurs in adult patients with diabetes mellitus. Today, the literature describes about 80 cases of this disease. The authors, who pay special attention to this pathology, point to its severity and note that 
often life-threatening complications occur with emphysematous pyelonephritis. All cases of emphysematous pyelonephritis were observed in adult patients. The process is usually onesided. There is no unified point of view on the treatment of emphysematous pyelonephritis $[1,6]$. Most authors believe that conservative therapy for this disease should be short-term, and if it is ineffective, nephrectomy should be used. Drainage of the kidney is recommended only in severely weakened patients with significant operational risk. It should be noted that some conditions, such as diabetes mellitus, urinary obstruction and immunodeficiency, predispose to the development of this disease. $[1,4,7,8$,$] .$

Escherichia coli, pseudomonas and proteus can lead to the development of emphysematous inflammation in the kidneys. Bacteria enter the kidney tissue when the urinary tract is clogged with a stone, kidney abscess or hematogenous in the presence of diabetes mellitus. Localization distinguishes between unilateral and bilateral emphysematous pyelonephritis. Women get sick more often than men. The lethality of this pathology is $60-80 \%[2,3,5,6$,$] .$

\section{RESEARCH METHOD}

In 2018, two patients with the pathology of emphysematous pyelonephritis were admitted in the emergency urology department of Fergana Medical Institute. The first patient was admitted to the emergency urology department on February 3rd, 2018 with complaints of fever, chills, weakness, malaise, dry mouth, nausea, vomiting, pain in the left abdomen. The following are the results from the initial examination and sampling:
General blood test: HB-109.0 g / I, erythrocytes

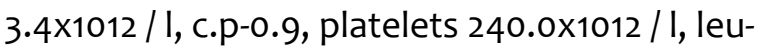
$10.3 \times 109 /$ l, ESR-38mm / h.

General urine analysis: protein-0.120 g / I, epithelium 2-3 in the field of view, leukocytes completely, erythrocytes unchanged 14-15, bacteria ++++ , mucus + .

Biochemical blood test: Total protein- $61.8 \mathrm{~g} / \mathrm{L}$, ALT 15.6 U / L, AST 14.6 U / L, Total bilirubin 14 mmol / L, bound bilirubin $2 \mathrm{mmol} / \mathrm{L}$, free bilirubin $12 \mathrm{mmol} / \mathrm{L}$, urea-5.8 $\mathrm{mmol} / \mathrm{l}$, glucose $4.4 \mathrm{mmol} / \mathrm{I}$. In the course of treatment, the indices of total protein decreased to $52.0 \mathrm{~g} / \mathrm{l}$.

\section{Instrumental Analysis - Patient 1}

Ultrasound of the kidneys: Left kidney $130 \mathrm{x}$ $60 \mathrm{~mm}$, TPP is not detected, Right kidney $155 \mathrm{x}$ $60 \mathrm{~mm}$, TPP-20mm. MSCT conclusion: Leftsided emphysematous pyelonephritis, paranephritis on the left, pyonephrosis on the left, lymphadenopathy of the abdominal space on the left. As a result of a thorough examination, the diagnosis was made: Complicated form of urinary tract infection. Acute pyelonephritis on the left, complicated by emphysematous pyelonephritis, acute paranephritis on the left, pyonephrosis on the left. Urosepsis. Hyperthermic syndrome.

Concomitant: Diabetes mellitus type II, severe course.

In the preoperative period, in order to prepare the patient for surgery, antibacterial therapy was prescribed (IV generation cephalosporins, III generation fluoroquinolones), infusion therapy was carried out to improve blood rheology (rheosorbilact, Ringer's solution, sodium chloride solution $0.9 \%$, sodium bicarbonate solution 4\%); symptomatic therapy with anesthetic and hypothermic purpose 
NSAIDs (diclofenac solution), antispasmodics (no-spa 2.0, platifillin $0.2 \%-1.0$ ). In order to regulate the protein balance, solutions of amino acids (infesol, selemin) were used, in the preoperative period, one-large fresh frozen plasma was transfused in an amount of $480 \mathrm{ml}$.

On March 5th, the patient underwent a surgery: posterior lumbotomy access according to Fedorov on the left. Nephrectomy on the left, debridement and drainage of the retroperitoneal space on the left.

In the postsurgical period, the patient continued adequate antibacterial and infusion therapy, transfused single-sized erythrocyte mass o (I) RH + in the amount of 1 liter $926 \mathrm{ml}$ and fresh frozen plasma in the amount of 4 liters $402 \mathrm{ml}$. In addition, In the postsurgical period, there was a decrease in total protein to $52.0 \mathrm{~g} / \mathrm{l}$. Blood tests were repeated over time: HB-107.0 g / I, erythrocytes 3.4×1012 / I, c.p 0.9, leukocytes -10.0x109 / I, ESR 8mm / hour. General urine analysis: protein-0.055g / I, single epithelium, leukocytes 3-4 in the field of view, erythrocytes unchanged 6-8 Biochemical blood test: total protein $62.0 \mathrm{~g} / \mathrm{l}$, ALT $10.0 \mathrm{U} / \mathrm{L}$, AST14.0 U / L, total bilirubin $12 \mathrm{mmol} / \mathrm{l}$, bound bilirubin $2 \mathrm{mmol} / \mathrm{l}$, free bilirubin $10 \mathrm{mmol} / \mathrm{l}$, urea-18.4 mmol / L, glucose $10.8 \mathrm{mmol} / \mathrm{L}$.

During 32 days of hospitalization, the general condition of the patient improved, insurance drains were removed and in a satisfactory condition was discharged for further observation by a urologist at the place of residence.

A similar case was observed in the second patient, who was transferred from the intensive care unit of the local branch of the emergency center to the intensive care unit of the Fergana Medical Institute in serious condition with complaints of fever up to 39 Celsius, dry mouth, nausea, weakness, malaise, bursting pains in the left abdomen and the lumbar region on the left. From the anamnesis, an increase in body temperature up to 39-40 Celsius is observed within a month.

Upon examination, the patient is severely emaciated, the general condition is serious, the skin is pale in color. In the left half of the lumbar region, the locally skin integuments are sharply tense. Laboratory tests: General blood count HB 91.0 g / I, erythrocytes 3.1 x1012 / l, c.p-0.9, leukocytes 15.5 x109/ I, ESR-8 mm/hour.

General urine analysis: protein-0.100 g / l, epithelium 1-2, leukocytes completely, erythrocytes unchanged 5-6, bacteria ++, biochemical blood test: total protein $52.8 \mathrm{~g} / \mathrm{I}$, ALT-36.0U / L, AST-AST- 18.0U / L, urea-19.8 $\mathrm{mmol} / \mathrm{l}$, glucose $13.8 \mathrm{mmol} / \mathrm{l}$.

\section{Instrumental analysis - Patient 2}

Ultrasound: in the perirenal space on the left, a large amount of purulent contents with the presence of gas. The left kidney is $100 \times 45 \mathrm{~mm}$, TPP is $13-14 \mathrm{~mm}$, the kidney is displaced up to the diaphragm. The presence of fluid in the left pleural sinus is 1.2 liters. Diagnosed with Complicated form of urinary tract infection. Acute emphysematous pyelonephritis on the left. Total purulent paranephritis on the left. Urosepsis. Hyperthermic syndrome. Concomitant: Transient renal failure. Uremic encephalopathy. Moderate anemia. Cachexia.

In the preoperative period, the patient was transfused with one-group $\mathrm{O}(\mathrm{I}) \mathrm{RH}+$ fresh frozen plasma in an amount of $480 \mathrm{ml}$, and adequate intensive antibiotic therapy was administered. 
On February 8th, 2018 the patient underwent surgery: Posterior lumbotomy access according to Fedorov on the left, about 2.5 liters of pus were evacuated. Left nephrectomy. During the operation, the patient was transfused with a single-sized erythrocyte mass in the amount of 1 liter. After the operation, the patient was transferred to the intensive care unit, where he received appropriate intensive therapy for 4 days. Subsequently, the Department of Emergent Urology continued antibacterial therapy (Ceftriaxone, Cefoperazone-sulbactam, Levofloxacin 500mg-100.0). Continued infusion therapy (Rheosorbilact, $0.9 \%$ sodium chloride solution, $4 \%$ sodium bicarbonate solution, Ringer's solution), antifungal drugs (fluconazole-100.0), NSAIDs (diclofenac solution). Infusion therapy continued in order to normalize blood rheology, transfusion of single-large erythrocyte mass in the amount of 1 liter $940 \mathrm{ml}$, fresh frozen plasma in the amount of 7 liters $073 \mathrm{ml}$. Every day, the postoperative wound was sanitized with Dekasan solution. The patient was treated in the department for 32 days and was discharged in satisfactory condition for further observation by the urologist at the place of residence.

\section{CONCLUSION}

1. Timely diagnosis and operative tactics, adequate treatment and postoperative care led to the preservation of life and improvement of the patient's condition.

2. In order to prevent complications in patients with diabetes mellitus, dispensary observation with control of glucose levels, urine and blood tests is necessary
1. Kukhtevich A.V. Gordovskaya N.B. Acute pyelonephritis // BC. Mother and child. 2018. - No. 23. - P. 5.

2. Lopatkin N.A., Pugachev A.G., Apolikhin O.I. Urology. - M .: Medicine, 2002 -- 348 p.

3. International classification of diseases from 2010 // ICD-10 Version: 2010. [Electronic resource].

4. Russian clinical guidelines. Urology // Russian Association of Urologists. - M., 2016 --- S. 429.

5. Chung V.Y., Tai C.K., Fan C.W., Tang C.N. Severe acute pyelonephritis: a review of clinical outcome and risk factors for mortality // Hong. Kong. Med. J. - 2014. - Vol. 20, No. 4. - P. 285-9.

6. FDA updates warnings for fluoroquinolone antibiotics // FDA News Release. [Electronic resource].

7. Lu Y.C., Chiang B.J., Pong Y.H., et al. Emphysematous pyelonephritis: clinical characteristics and prognostic factors // Int. J. Urol. - 2014. - Vol. 21, No. 3. - P. 277-82.

8. Mc Lellan L.K., Hunstad D.A. Urinary Tract Infection: Pathogenesis and Outlook // Trends. Mol. Med. - 2016. - Vol. 22, No. 11. - P. 946-57.

\section{REFERENCES}

